\title{
An ethnobotanical study of the useful and edible plants of İzmit
}

\author{
Çağla Kızılarslan1, Neriman Özhatay²
}

ABSTRACT: An ethnobotanical study was carried out in İzmit province (Northwest Turkey). During this study, 145 specimens were collected from the area. Informations about plants such as their usages and used parts were recorded. The stud y revealed that, 96 plant taxa belonging to 42 families had ethnobotanical usages in this area. Among these 96 plant taxa, 60 taxa (51 wild, 9 cultivated) were used as edibles and 64 taxa (51 wild, 13 cultivated) were used for different purposes. The results of our study show that even in countrys that are situated in close proximity to metropolitan cities, the ethnobotanical usages of plants are still alive.

KEY WORDS: Ethnobotany, Turkey, İzmit

\section{INTRODUCTION}

In terms of plant diversity Turkey is one of the richest countries in the world. The Turkish flora is estimated to contain more than 10.000 species of vascular plants of which about 3.034 (approximately $34 \%$ ) are endemic (1-6).

For a long time plants have played very important role for human life. As is the case with elsewhere in the world, Turkish people have utilized plants for a long time as medicinal, food, fuel and dye, as well as for ornamentation, agricultural tools, furniture and construction materials. Ethnobotanical studies have been carried out in Turkey since the early years of the $19^{\text {th }}$ century (7).

The aim of this study is to collect information about the ethnobotanical usages in İzmit province (Northwest Turkey) before they are completely lost. In this paper, priority is given to the description of useful and edible plants in İzmit province (Northwest Turkey).

\section{MATERIAL AND METHODS}

Our research area, İzmit, is situated in Marmara Region in Northwest Turkey (Figure 1). İzmit is the centre province of Kocaeli and it has an area of $974 \mathrm{~km}^{2}$ and its population is 373.034 . Izmit is a coast county which is established between the most important Asia and Europe transition line. It has close proximity to İstanbul metropolitan

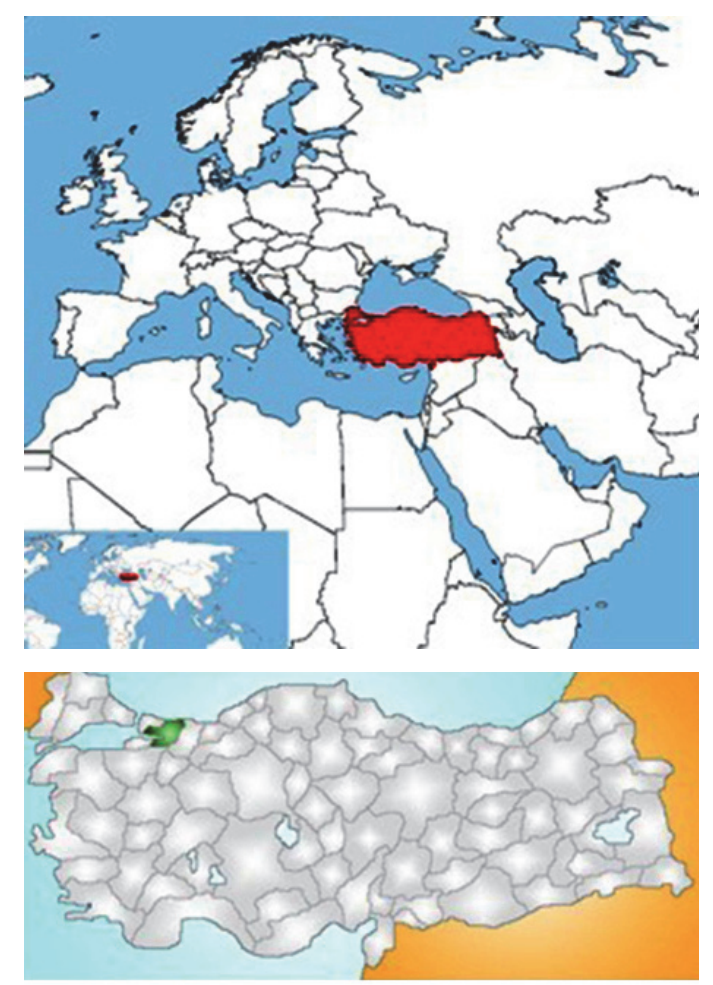

FIGURE 1. Study area: İzmit district, Turkey

city. The economy in the county mostly based on industry. Because of this, the majority of the population consists of people all around the Turkey and also immigrants from Balkans and Caucasus.
AFFILIATIONS

1Bezmialem Vakif

Üniversitesi, Eczacılık

Fakültesi Farmasötik Botanik

Anabilim Dalı, İstanbul,

Türkiye

2istanbul Üniversitesi,

Eczacılık Fakültesi

Farmasötik Botanik Anabilim Dalı, İstanbul, Türkiye

\section{CORRESPONDENCE}

Çă̆la Kızılarslan

E-mail:

c.kizilarslan@gmail.com

Received:

08.05.2012

Revision:

21.06.2012

Accepted:

22.06.2012 
This study is a part of a master thesis named "An Ethnobotanical Survey in The South Part of İzmit Gulf" (8). The field works of the study were carried out between April 2006-September 2007. During the research, 9 municipalities and 15 villages were visited and 145 specimens were collected. The informations for these plants, such as their usages and used parts were recorded to "Ethnobotanical data forms". Information was collected from both the elder and the young local people through interviews. The plants were collected with the help of the informants. Taxonomical determination of the collected specimens was made by using "Flora of Turkey and the East Aegean Islands" (1-3) and "Flora of Europaea" (9). The voucher specimens were kept in the Herbarium of the Faculty of Pharmacy, İstanbul University (ISTE). Scientific names of plant species were identified according to the International Plant Names Index (http:/ / www.ipni.org).
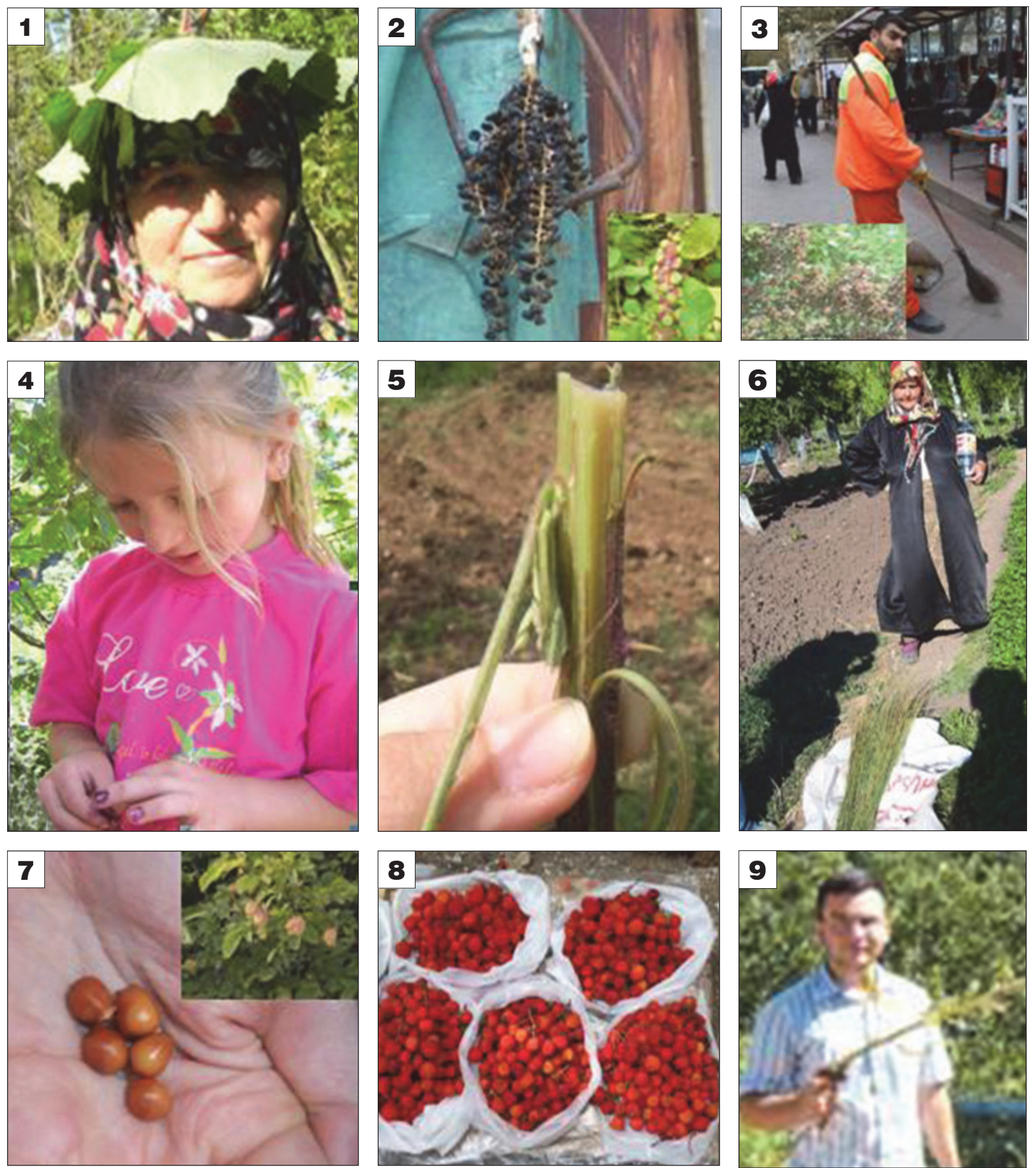

FIGURE 2. (1) Usage of Petasites hybridus leaves as hat, (2) Dried Phytolacca americana fruits for colouring cabbage pickle to pink, (3) Erica arborea as broom, (4) Sambucus nigra fruits as nail polish, (5) Rubus sanctus stem as food, (6) Juncus inflexus for wattling, (7) Euonymus latifolius subsp. latifolius seeds for making bead, (8) Arbutus unedo fruits in the bazaar, (9) Verbascum speciosum as a gun in children's play. 
TABLE 1. Ethnobotanical usages of plants in Izmit province.

\begin{tabular}{|c|c|c|c|}
\hline $\begin{array}{l}\text { Scientific name } \\
\text { Family name (Voucher specimen number) }\end{array}$ & Local names & Used parts & Uses \\
\hline $\begin{array}{l}\text { Alcea setosa Alef. } \\
\text { Malvaceae (ISTE } 84 \text { 029, ISTE } 84 \text { 176) }\end{array}$ & $\begin{array}{l}\text { Hatmi, Hatmiçiçeği, } \\
\text { Karafatma, Yabanifatmagül }\end{array}$ & Leaf & $\begin{array}{c}\text { For hair care } \\
\text { Cleaning the house against dust }\end{array}$ \\
\hline $\begin{array}{l}\text { Amaranthus hybridus L. } \\
\text { Amaranthaceae (ISTE } 84 \text { 185) }\end{array}$ & Iştır, Karagözmancarı, Telliıştır & Leaf and young stem & Cooked as a meal and for pastry \\
\hline \multirow[t]{2}{*}{$\begin{array}{l}\text { Arbutus unedo L. } \\
\text { Ericaceae (ISTE } 84 \text { 051) }\end{array}$} & $\begin{array}{l}\text { Andrana, Dağyemişi, Kocakarıyemişi, } \\
\text { Kocayemiş, Ormançileği, Piyadin }\end{array}$ & Fruit & Eaten fresh or as jam \\
\hline & & Wood & As fuel \\
\hline $\begin{array}{l}\text { Arum italicum Mill. } \\
\text { Araceae (ISTE } 84 \text { 151) }\end{array}$ & $\begin{array}{l}\text { Çiçekotu, Tirşik, Yılanbıçağı, Yılanotu, } \\
\text { Yılansoğanı, Yılanyastığı, Yılanzehiri, Zehirotu }\end{array}$ & Leaf & $\begin{array}{l}\text { Boiled a few times for a long time in several } \\
\text { waters to detoxificate because it contains } \\
\text { poisinous alkaloids. "Tirşik soup" is made. }\end{array}$ \\
\hline \multirow[t]{2}{*}{$\begin{array}{l}\text { Bellis perennis L. } \\
\text { Asteraceae (ISTE } 84 \text { 121) }\end{array}$} & Beyazpapatya & Leaf & Cooked with vegetables \\
\hline & & Capitulum & As tea \\
\hline $\begin{array}{l}\text { ^Brassica oleracea L. var. acephala DC. } \\
\text { Brassicaceae (ISTE } 84 \text { 119) }\end{array}$ & Karalahana, Pali & Leaf & Cooked as meal, soup and farci \\
\hline $\begin{array}{l}\text { Calepina irregularis Thell. } \\
\text { Brassicaceae (ISTE } 84 \text { 099) }\end{array}$ & - & Leaf & Boiled and eaten as salad \\
\hline $\begin{array}{l}\text { Capsella bursa-pastoris (L.) Medik. } \\
\text { Brassicaceae (ISTE } 84 \text { 065) }\end{array}$ & $\begin{array}{c}\text { Çobançantası, Derelahanası, Kazayağı, } \\
\text { Kazbağsı, Kuşayağı }\end{array}$ & Leaf & Eaten as salad and cooked for pastry \\
\hline \multirow[t]{2}{*}{$\begin{array}{l}\text { Cardamine hirsuta L. } \\
\text { Brassicaceae (ISTE } 84 \text { 095) }\end{array}$} & - & Leaf & $\begin{array}{l}\text { Boiled and eaten as salad, } \\
\text { cooked with onion as a meal }\end{array}$ \\
\hline & & Seed & Boiled and eaten \\
\hline \multirow[t]{2}{*}{$\begin{array}{l}\text { *Castanea sativa Mill. } \\
\text { Fagaceae (ISTE } 84 \text { 063) }\end{array}$} & Kestane, Kestane ağacı & Wood & As fuel \\
\hline & & Flower & $\begin{array}{l}\text { As beeplant for getting } \\
\text { "Kestane Balı (Chestnut honey)" }\end{array}$ \\
\hline $\begin{array}{l}\text { *Cerasus avium Moench } \\
\text { Rosaceae (ISTE } 84 \text { 147) }\end{array}$ & Kiraz & Fruit & Eaten fresh \\
\hline \multirow{2}{*}{$\begin{array}{l}\text { Chaerophyllum byzantinum Boiss. } \\
\text { Apiaceae (ISTE } 84 \text { 044) }\end{array}$} & \multirow[t]{2}{*}{ Çarşır, Çarşırotu, Yoğurtotu } & Leaf and stem & $\begin{array}{l}\text { Cooked with onion as a meal } \\
\text { As fodder }\end{array}$ \\
\hline & & Stem & As pickle \\
\hline $\begin{array}{l}\text { Chenopodium album L. subsp. album var. } \\
\text { album } \\
\text { Chenopodiaceae (ISTE } 84 \text { 153) }\end{array}$ & $\begin{array}{l}\text { Cimel, Evlidaotu, Güllüotu, } \\
\text { Küllümancar, Tavukotu }\end{array}$ & Leaf and young stem & $\begin{array}{l}\text { Boiled and eaten as a meal or salad, } \\
\text { cooked for pastry }\end{array}$ \\
\hline $\begin{array}{l}\text { Cichorium intybus L. } \\
\text { Asteraceae (ISTE } 84 \text { 047) }\end{array}$ & Hindiba, Mavihindiba, Radika, Sakızotu & Leaf and stem & Boiled and eaten as salad, cooked for pastry \\
\hline $\begin{array}{l}\text { Cirsium creticum d'Urv. subsp. creticum } \\
\text { Asteraceae (ISTE } 84 \text { 123) }\end{array}$ & Eşekçalısı, Eşekdikeni & Stem & $\begin{array}{l}\text { After bark is peeled, cooked as } \\
\text { a meal or eaten fresh }\end{array}$ \\
\hline $\begin{array}{l}\text { Cistus creticus L. } \\
\text { Cistaceae (ISTE } 84 \text { 028, ISTE } 84 \text { 050) }\end{array}$ & İstifiza, Karağan, Laden & Branch with leaf & As tea \\
\hline $\begin{array}{l}\text { Convolvulus arvensis L. } \\
\text { Convolvulaceae (ISTE } 84 \text { 112) }\end{array}$ & Babootu, Leksiotu, Tosbağaotu & $\begin{array}{l}\text { Leaf } \\
\text { Aerial part }\end{array}$ & $\begin{array}{l}\text { Cooked with vegetables } \\
\text { As fodder }\end{array}$ \\
\hline $\begin{array}{l}\text { Convolvulus betonicifolius Mill. subsp. } \\
\text { betonicifolius } \\
\text { Convolvulaceae (ISTE } 84 \text { 149) }\end{array}$ & Leksiotu & Aerial part & As fodder for cow \\
\hline \multirow{2}{*}{$\begin{array}{l}{ }^{\star} \text { Cornus mas L. } \\
\text { Cornaceae (ISTE } 84 \text { 089) }\end{array}$} & \multirow{2}{*}{ Kızılcık } & Fruit & Eaten as jam, marmalade and tarhana soup \\
\hline & & Leaf & For colouring henna \\
\hline *Cydonia oblonga Mill. & \multirow{2}{*}{ Ayva } & Fruit & Eaten fresh or as jam \\
\hline Rosaceae (ISTE 84 170) & & Leaf & For colouring henna and dying cloth \\
\hline $\begin{array}{l}\text { Cynodon dactylon (L.) Pers. var. dactylon } \\
\text { Poaceae (ISTE } 84 \text { 053) }\end{array}$ & Ayrıkotu, Beygirotu & Whole plant & As fodder \\
\hline $\begin{array}{l}\text { Datura stramonium L. } \\
\text { Solanaceae (ISTE } 84 \text { 080) }\end{array}$ & Afyonotu, Eşekdikeni, Eşekotu & $\begin{array}{c}\text { Fruit } \\
\text { Leaf, seed }\end{array}$ & $\begin{array}{l}\text { As comb in children's play } \\
\text { As cigarette }\end{array}$ \\
\hline $\begin{array}{l}\text { Dipsacus laciniatus L. } \\
\text { Dipsacaceae (ISTE } 84 \text { 179) }\end{array}$ & Eşekkengeri & Aerial part & Treating mouth sores in donkey \\
\hline $\begin{array}{l}\text { Erica arborea L. } \\
\text { Ericaceae (ISTE } 84 \text { 129, ISTE } 84 \text { 141) }\end{array}$ & Süpürgeotu & Aerial part & As broom and fuel \\
\hline $\begin{array}{l}\text { Erodium malacoides (L.) L'Hér. } \\
\text { Geraniaceae (ISTE } 84 \text { 161) }\end{array}$ & - & Branch with leaf & Cooked with vegetables \\
\hline $\begin{array}{l}\text { Euonymus latifolius Mill. subsp. latifolius } \\
\text { Celastraceae (ISTE } 84 \text { 193) }\end{array}$ & - & Seed & For making bead \\
\hline
\end{tabular}


TABLE 1 CONTINUED. Ethnobotanical usages of plants in Izmit province.

\begin{tabular}{|c|c|c|c|}
\hline $\begin{array}{l}\text { Scientific name } \\
\text { Family name (Voucher specimen number) }\end{array}$ & Local names & Used parts & Uses \\
\hline \multirow{2}{*}{$\begin{array}{l}\text { *Ficus carica L. subsp. carica } \\
\text { Moraceae (Observation) }\end{array}$} & \multirow{2}{*}{ İncir, Yemiş, Yemişen } & Fruit & Eaten fresh or as jam \\
\hline & & Leaf & Added to anchovy \\
\hline $\begin{array}{l}\text { Geranium asphodeloides Sibth. \& Sm. } \\
\text { subsp. asphodeloides } \\
\text { Geraniaceae (ISTE } 84 \text { 166) }\end{array}$ & - & Branch with leaf & Cooked with vegetables \\
\hline $\begin{array}{l}\text { Geranium purpureum Vill. } \\
\text { Geraniaceae (ISTE } 84 \text { 163) }\end{array}$ & Hıdır, Yabanihıdır & Branch with leaf & Cooked with vegetables and for pastry \\
\hline $\begin{array}{l}\text { Helleborus orientalis Lam. } \\
\text { Ranunculaceae (ISTE } 84 \text { 126) }\end{array}$ & Bohça, Bohçaotu, Çöpleme, Çöpotu & Leaf, rhizome & Against diarrhea and chill in animals \\
\hline \multirow{2}{*}{$\begin{array}{l}\text { Heracleum platytaenium Boiss. } \\
\text { Apiaceae (ISTE } 84 \text { 171) }\end{array}$} & \multirow{2}{*}{$\begin{array}{l}\text { Havlan, Halvanotu, Hometi, Kekrer, } \\
\text { Romati, Yabanlahanası }\end{array}$} & Stem and stalk & As pickle \\
\hline & & Leaf & As fodder for cow \\
\hline \multirow{2}{*}{$\begin{array}{l}\text { *Juglans regia L. } \\
\text { Juglandaceae (ISTE } 84 \text { 120) }\end{array}$} & \multirow{2}{*}{ Ceviz } & Seed & Eaten fresh \\
\hline & & Pericarp & Tea as panacea \\
\hline $\begin{array}{l}\text { Juncus inflexus L. } \\
\text { Juncaceae (ISTE } 84 \text { 045) }\end{array}$ & Sazotu & Stem & $\begin{array}{c}\text { For wattling and tying } \\
\text { Petroselinum sativum bunches }\end{array}$ \\
\hline \multirow{2}{*}{$\begin{array}{l}\text { Lamium purpureum L. var. purpureum } \\
\text { Lamiaceae (ISTE } 84 \text { 103) }\end{array}$} & \multirow{2}{*}{$\begin{array}{l}\text { Arıotu, Ballıbaba, } \\
\text { Balotu, Petekotu }\end{array}$} & Aerial part & Cooked with vegetables and for pastry \\
\hline & & Flower & Children aspirate its nectar \\
\hline $\begin{array}{l}\text { Lathyrus undulatus Boiss. } \\
\text { Fabaceae (ISTE } 84 \text { 077) }\end{array}$ & Ladir & Aerial part & As fodder \\
\hline \multirow{2}{*}{$\begin{array}{l}\text { Laurocerasus officinalis M. Roem. } \\
\text { Rosaceae (ISTE } 84 \text { 115) }\end{array}$} & \multirow{2}{*}{ Karamiş, Karayemiş } & Fruit & Eaten fresh \\
\hline & & Seed & As tea \\
\hline $\begin{array}{l}\text { Laurus nobilis L. } \\
\text { Lauraceae (ISTE } 84 \text { 048) }\end{array}$ & Defne, Define, Depne & Leaf & $\begin{array}{l}\text { As spice in meat and fish } \\
\text { Used for hair care }\end{array}$ \\
\hline \multirow{2}{*}{$\begin{array}{l}\text { Malva nicaeensis All. } \\
\text { Malvaceae (ISTE } 84 \text { 136) }\end{array}$} & \multirow{2}{*}{$\begin{array}{l}\text { Ebegömeci, Ebegömeç, } \\
\text { Ebegümeci }\end{array}$} & Leaf & $\begin{array}{l}\text { Cooked as a meal } \\
\text { Used for decreasing Urtica sp. prickles }\end{array}$ \\
\hline & & Fruit & For making necklace and bracelet \\
\hline $\begin{array}{l}\text { Malva sylvestris L. } \\
\text { Malvaceae (ISTE } 84 \text { 092) }\end{array}$ & $\begin{array}{l}\text { Ebegömeç, Ebegümeci, } \\
\text { Molaşa, Molaşaotu }\end{array}$ & Stem and leaf & $\begin{array}{l}\text { Cooked as a meal and } \\
\text { for pastry }\end{array}$ \\
\hline \multirow{2}{*}{$\begin{array}{l}\text { Medicago arabica (L.) Huds. } \\
\text { Fabaceae (ISTE } 84 \text { 030) }\end{array}$} & \multirow{2}{*}{ Yonca } & Leaf & Cooked with vegetables \\
\hline & & Aerial part & As fodder \\
\hline \multirow{2}{*}{$\begin{array}{l}\text { Melissa officinalis L. subsp. } \\
\text { altissima (Sm.) Arcang. } \\
\text { Lamiaceae (ISTE } 84 \text { 070, ISTE } 84 \text { 073) }\end{array}$} & \multirow{2}{*}{$\begin{array}{c}\text { Oğulotu, Saçkıran, } \\
\text { Yabanısırganı, Yabanidereotu }\end{array}$} & Leaf & Boiled as salad, cooked as a meal \\
\hline & & Aerial part & $\begin{array}{l}\text { As tea for bracing (Decoction with } \\
\text { Urtica sp. ve Malva sp. leaves) }\end{array}$ \\
\hline $\begin{array}{l}\text { Mentha longifolia Huds. subsp. typhoides } \\
\text { (Briq.) Harley var. typhoides } \\
\text { Lamiaceae (ISTE } 84 \text { 059) }\end{array}$ & $\begin{array}{l}\text { Nane, Yabaninane, } \\
\text { Yabannanesi }\end{array}$ & Leaf & As spice \\
\hline $\begin{array}{l}\text { Mentha spicata L. subsp. spicata } \\
\text { Lamiaceae (ISTE } 84 \text { 187) }\end{array}$ & Nane, Yabaninane & Leaf & As spice \\
\hline \multirow{2}{*}{$\begin{array}{l}\text { *Mespilus germanica L. } \\
\text { Rosaceae (ISTE } 84 \text { 113) }\end{array}$} & \multirow{2}{*}{ Döngel, Muşmula } & Fruit & Eaten fresh or as jam \\
\hline & & Leaf & For colouring henna \\
\hline $\begin{array}{l}\text { *Morus alba L. } \\
\text { Moraceae (ISTE } 84 \text { 137) }\end{array}$ & Dut, Mora & Fruit & Eaten fresh or as jam \\
\hline \multirow{2}{*}{$\begin{array}{l}\text { *Morus nigra L. } \\
\text { Moraceae (Observation) }\end{array}$} & \multirow[t]{2}{*}{ Ekşi karadut, Karadut } & Fruit & $\begin{array}{l}\text { Eaten fresh or as jam } \\
\text { Children paint their hands and faces }\end{array}$ \\
\hline & & Leaf & Cooked with vegetables \\
\hline \multirow{2}{*}{$\begin{array}{l}\text { Muscari neglectum Ten. } \\
\text { Liliaceae (ISTE } 84 \text { 071) }\end{array}$} & \multirow{2}{*}{ Kargasoğanı, Saçkıran } & Leaf & For plaiting hairs in children's play \\
\hline & & Flower & For painting the egg to blue-purple \\
\hline $\begin{array}{l}\text { Oenanthe pimpinelloides L. } \\
\text { Apiaceae (ISTE } 84 \text { 142) }\end{array}$ & $\begin{array}{l}\text { Kazayağı, Kazayak, Kazbacağı, Kazıyak, } \\
\text { Kazyağı, Kazyakotu, Yabanimaydanoz }\end{array}$ & Leaf & $\begin{array}{l}\text { Boiled as pickle and salad, cooked as a meal } \\
\text { with rice and egg }\end{array}$ \\
\hline $\begin{array}{l}\text { Origanum vulgare L. subsp. } \\
\text { hirtum (Link) letsw. } \\
\text { Lamiaceae (ISTE } 84 \text { 067) }\end{array}$ & Kekik, Kekikotu, Köfteotu & Branch with leaf & As spice \\
\hline $\begin{array}{l}\text { Ornithogalum sigmoideum Freyn \& Sint. } \\
\text { Liliaceae (ISTE } 84 \text { 087) }\end{array}$ & Çiğdemçiçeği, Kargasarımsağı & Leaf, stem, bulb & Cooked with rice \\
\hline $\begin{array}{l}\text { Paliurus spina-christi Mill. } \\
\text { Rhamnaceae (ISTE } 84 \text { 172) }\end{array}$ & $\begin{array}{l}\text { Avanaktamisi, Dikenliçalı, } \\
\text { Karaçalı, Karaçalıdikeni }\end{array}$ & Aerial part & For making fence \\
\hline Papaver rhoeas L. & Gelincik Gelincikotu Kukumavotu Nünü & Leaf & Cooked as a meal and for pastry \\
\hline Papaveraceae (ISTE 84 148, ISTE 84 183) & Gelinclk, Gelinclkotu, Kukumavotu, Ivunu & Petal & Boiled as sherbet \\
\hline $\begin{array}{l}\text { Petasites hybridus (L.) G.Gaertn., } \\
\text { B.Mey. \& Scherb. } \\
\text { Asteraceae (ISTE } 84 \text { 116) }\end{array}$ & $\begin{array}{l}\text { Ayıkulağı, Farafla, Kabakulakotu, } \\
\text { Kabalak, Şemsiyeotu }\end{array}$ & Leaf & $\begin{array}{c}\text { For making hat and umbrella with } 2 \text { big leaves } \\
\text { while working in gardens } \\
\text { As fodder and for wound healing in animal } \\
\text { diseases }\end{array}$ \\
\hline
\end{tabular}


TABLE 1 CONTINUED. Ethnobotanical usages of plants in Izmit province.

\begin{tabular}{|c|c|c|c|}
\hline $\begin{array}{l}\text { Scientific name } \\
\text { Family name (Voucher specimen number) }\end{array}$ & Local names & Used parts & Uses \\
\hline Phytolacca americana L. & \multirow{2}{*}{ Kuşüzümü } & Leaf & Cooked with vegetables \\
\hline Phytolaccaceae (ISTE 84 076) & & Fruit & For colouring cabbage pickle to pink \\
\hline $\begin{array}{l}\text { Plantago major L. subsp. major } \\
\text { Plantaginaceae (ISTE } 84 \text { 127) }\end{array}$ & $\begin{array}{l}\text { Balazağva, Damarlıt, Damarotu, } \\
\text { Kırksinirotu, Sinirliot, Sinirotu }\end{array}$ & Leaf & Eaten as meal and stuffing \\
\hline $\begin{array}{l}\text { Prunus spinosa L. subsp. } \\
\text { dasyhylla (Schur) Domin } \\
\text { Rosaceae (ISTE } 84 \text { 064) }\end{array}$ & Çakaleriği, Güvem, Yabanierik & Fruit & Eaten fresh or as jam \\
\hline $\begin{array}{l}\text { Pteridium aquilinum (L.) Kuhn } \\
\text { Hypolepidaceae (ISTE } 84 \text { 056) }\end{array}$ & Eğrelti, Ifteri & Aerial part & $\begin{array}{l}\text { As carpet in children's play, as fodder and } \\
\text { for dust inhibiting in barns }\end{array}$ \\
\hline $\begin{array}{l}\text { Ranunculus constantinopolitanus d'Urv. } \\
\text { Ranunculaceae (ISTE } 84 \text { 038) }\end{array}$ & Sakızotu & Petal & For colouring chewing gum in children's play \\
\hline $\begin{array}{l}\text { Ranunculus ficaria L. subsp. } \\
\text { ficariiformis Rouy\&Foucaud } \\
\text { Ranunculaceae (ISTE } 84 \text { 091) }\end{array}$ & Yağlıot & Leaf & Boiled as salad, cooked as a meal \\
\hline Raphanus raphanistrum L. & Karamancar, Karaturp, & Leaf and stem & Boiled as salad, cooked as a meal \\
\hline Brassicaceae (ISTE 84 078) & Turpotu, Yabaniturpotu & Stem & As pickle \\
\hline \multirow{2}{*}{$\begin{array}{l}\text { Rhodendron ponticum L. subsp. ponticum } \\
\text { Ericaceae (ISTE } 84 \text { 055) }\end{array}$} & \multirow{2}{*}{$\begin{array}{l}\text { Avu, Komargülü, } \\
\text { Ormangülü }\end{array}$} & Flower & $\begin{array}{l}\text { As decorative and beeplant for getting } \\
\text { "Deli Bal (Poisonous honey)" }\end{array}$ \\
\hline & & Aerial part & As fuel \\
\hline \multirow{2}{*}{$\begin{array}{l}\text { Rosa canina L. } \\
\text { Rosaceae (ISTE } 84 \text { 041, ISTE } 84 \text { 146) }\end{array}$} & \multirow{2}{*}{$\begin{array}{l}\text { Dikenbaşı, Köpekgülü, Kuşburnu, } \\
\text { Öküzgötü, Yabanigül }\end{array}$} & Fruit & Eaten fresh or as jam \\
\hline & & Petal & Eaten as panacea \\
\hline \multirow{2}{*}{$\begin{array}{l}\text { *Rosmarinus officinalis L. } \\
\text { Lamiaceae (ISTE } 84 \text { 118) }\end{array}$} & \multirow{2}{*}{ Biberiye } & Leaf & As spice \\
\hline & & Whole plant & As mosquito repellent \\
\hline \multirow{2}{*}{$\begin{array}{l}\text { Rubus canescens DC. var. canescens } \\
\text { Rosaceae (ISTE } 84 \text { 144) }\end{array}$} & \multirow{2}{*}{$\begin{array}{l}\text { Böğürtlen, Diken, Hamdüspara, } \\
\text { Karamuk, Mora }\end{array}$} & Fruit & Eaten fresh or as jam \\
\hline & & Young shoot & $\begin{array}{l}\text { After bark is peeled, eaten or } \\
\text { cooked with vegetables }\end{array}$ \\
\hline \multirow{2}{*}{$\begin{array}{l}\text { Rubus sanctus Schreb. } \\
\text { Rosaceae (ISTE } 84 \text { 145) }\end{array}$} & \multirow{2}{*}{$\begin{array}{l}\text { Böğürtlem, Böğürtlen, Börtlen, Diken, } \\
\text { Dikenbaşı, Mora }\end{array}$} & Fruit & Eaten fresh or as jam \\
\hline & & Young shoot & $\begin{array}{c}\text { After bark is peeled, eaten or cooked with } \\
\text { vegetables }\end{array}$ \\
\hline $\begin{array}{l}\text { Rumex acetosella L. } \\
\text { Polygonaceae (ISTE } 84 \text { 046) }\end{array}$ & Ekşiotu, Kuzukulağı & Leaf & Eaten fresh or boiled as salad \\
\hline \multirow{2}{*}{$\begin{array}{l}\text { Rumex pulcher L. } \\
\text { Polygonaceae (ISTE } 84 \text { 159) }\end{array}$} & \multirow{2}{*}{$\begin{array}{l}\text { Çarşaf, Efelek, Efelik, Labada, Lapaza, } \\
\text { Mancar, Mancarotu, Pancarotu, Yapalak }\end{array}$} & Leaf & Eaten as meal and stuffing \\
\hline & & Fruit & Against cough in animals \\
\hline $\begin{array}{l}\text { Ruscus aculeatus L. var. aculeatus } \\
\text { Liliaceae (ISTE } 84 \text { 039) }\end{array}$ & Çalısüpürgesi, Kuşkondurmadikeni & Aerial part & As broom and in floriculture \\
\hline \multirow{2}{*}{$\begin{array}{l}\text { Ruscus hypoglossum L. } \\
\text { Liliaceae (ISTE } 84 \text { 068) }\end{array}$} & \multirow{2}{*}{ Aleksandra, Karamut } & Fruit & Eaten fresh \\
\hline & & Aerial part & As fodder \\
\hline $\begin{array}{l}\text { Salvia virgata Ait. } \\
\text { Lamiaceae (ISTE } 84 \text { 106, ISTE } 84 \text { 181) }\end{array}$ & Yağlısomara & Whole plant & As fodder \\
\hline \multirow{2}{*}{$\begin{array}{l}\text { Sambucus ebulus L. } \\
\text { Caprifoliaceae (ISTE } 84 \text { 184) }\end{array}$} & \multirow{2}{*}{$\begin{array}{l}\text { Lor, Lüver, Piran, Sultan, } \\
\text { Şahmelek, Şahmelekotu }\end{array}$} & Leaf & Decreasing Urtica sp. prickles \\
\hline & & Leaf, stem & $\begin{array}{l}\text { For chick diseases and } \\
\text { as acaricide in animals }\end{array}$ \\
\hline \multirow{2}{*}{$\begin{array}{l}\text { Sambucus nigra L. } \\
\text { Caprifoliaceae (ISTE } 84 \text { 180) }\end{array}$} & \multirow{2}{*}{$\begin{array}{l}\text { Lor, Lüver, Lüvor, Melikşah, } \\
\text { Piran, Piren, Sultan, Sultanotu, } \\
\text { Şahmelek, Şahmelik, Yiğidinotu }\end{array}$} & Leaf & $\begin{array}{l}\text { Putting over the tobacco bales to } \\
\text { make moisture }\end{array}$ \\
\hline & & Fruit & As nail polish in children's play \\
\hline \multirow{2}{*}{$\begin{array}{l}\text { Scrophularia scopolii Hoppe ex Pers. var. } \\
\text { scopolii } \\
\text { Scrophulariaceae (ISTE } 84 \text { 173) }\end{array}$} & \multirow[b]{2}{*}{ Ballıbaba, Süpürgelik } & Aerial part & As broom \\
\hline & & Flower & Children aspirate its nectar \\
\hline \multirow{2}{*}{$\begin{array}{l}\text { Senecio vulgaris L. } \\
\text { Asteraceae (ISTE } 84 \text { 134) }\end{array}$} & \multirow[t]{2}{*}{ Sütlüce } & Leaf & $\begin{array}{l}\text { Boiled, filtered the boiling water and } \\
\text { eaten as salad }\end{array}$ \\
\hline & & Aerial part & As fodder \\
\hline $\begin{array}{l}\text { Silybum marianum (L.) Gaertn. } \\
\text { Asteraceae (ISTE } 84 \text { 169) }\end{array}$ & Kocabaş & Stem, young shoot & $\begin{array}{l}\text { After bark is peeled, eaten fresh or } \\
\text { cooked with rice }\end{array}$ \\
\hline Smilax excelsa L. & Gıcırdakdikeni, Kuşevin, & Young shoot, leaf & Cooked as meal \\
\hline Liliaceae (ISTE 84 150, ISTE 84 186) & Zimilaçidikeni & Fruit & Chewing like gum in children's play \\
\hline $\begin{array}{l}\text { Solanum nigrum L. subsp. nigrum } \\
\text { Solanaceae (ISTE } 84 \text { 037, ISTE } 84 \text { 049) }\end{array}$ & $\begin{array}{c}\text { Köpekdomatesi, Tarlaüzümcüğü, } \\
\text { Üzümcük }\end{array}$ & Fruit & Eaten fresh \\
\hline $\begin{array}{l}\text { Sonchus asper (L.) Hill subsp. glaucescens } \\
\text { (Jord) Ball }\end{array}$ & Çallıcaotu, Özsütlü, Sütlen, & Stem, leaf & Eaten as salad, cooked with vegetables \\
\hline $\begin{array}{l}\text { (Jord.) Ball } \\
\text { Asteraceae (ISTE } 84 \text { 042, ISTE } 84 \text { 168) }\end{array}$ & Sütlük, Sütlüotu & Aerial part & As fodder \\
\hline $\begin{array}{l}\text { Sorghum halepense (L.) Pers. var. halepense } \\
\text { Poaceae (ISTE } 84 \text { 052) }\end{array}$ & Ekinotu, Mısırotu & Aerial part & As broom and fodder \\
\hline
\end{tabular}


TABLE 1 CONTINUED. Ethnobotanical usages of plants in Izmit province.

\begin{tabular}{|c|c|c|c|}
\hline $\begin{array}{l}\text { Scientific name } \\
\text { Family name (Voucher specimen number) }\end{array}$ & Local names & Used parts & Uses \\
\hline $\begin{array}{l}\text { Spartium junceum L. } \\
\text { Fabaceae (ISTE } 84 \text { 139) }\end{array}$ & Katırtırnağı & Aerial part & As broom, fuel, decorative \\
\hline $\begin{array}{l}\text { Stellaria media (L.) Vill. subsp. media } \\
\text { Caryophyllaceae (ISTE } 84 \text { 093) }\end{array}$ & $\begin{array}{l}\text { Arapsaçı, Bürümcek, Gıyşak, } \\
\text { Kulumcak, Kuşotu, Kuşyüreği }\end{array}$ & $\begin{array}{l}\text { Young shoot, } \\
\text { branch with leaf }\end{array}$ & Eaten as salad, cooked with rice or for pastry \\
\hline $\begin{array}{l}\text { Taraxacum scaturiginosum G.E. Haglund } \\
\text { Asteraceae (ISTE } 84 \text { 154) }\end{array}$ & Hindiba, Sarısütlü, Sütlüot & $\begin{array}{l}\text { Leaf } \\
\text { Stem }\end{array}$ & $\begin{array}{l}\text { Cooked as meal, boiled as salad } \\
\text { As whistle in children's play }\end{array}$ \\
\hline $\begin{array}{l}\text { Thymus longicaulis C. Presl subsp. } \\
\text { longicaulis var. subisophyllus (Borbás) Jalas } \\
\text { Lamiaceae (ISTE } 84 \text { 132) }\end{array}$ & Kekik & Leaf & As spice \\
\hline $\begin{array}{l}\text { Tilia argentea DC. } \\
\text { Tiliaceae (ISTE } 84 \text { 054) }\end{array}$ & Ihlamur & Wood & As fuel and decorative \\
\hline $\begin{array}{l}\text { Trachystemon orientalis (L.) G. Don } \\
\text { Boraginaceae (ISTE } 84 \text { 114) }\end{array}$ & $\begin{array}{l}\text { Çiçeklimancar, Hodan, Kaldırak, Kaldırek, } \\
\text { Kaldırik, Somara, Tomari, Tomara, Zılbıt }\end{array}$ & Stem, leaf & Cooked as meal \\
\hline $\begin{array}{l}\text { Trifolium constantinopolitanum Ser. } \\
\text { Fabaceae (ISTE } 84 \text { 040) }\end{array}$ & Üçkulakotu, Yonca & Aerial part & As fodder \\
\hline $\begin{array}{l}\text { Trifolium resupinatum L. var. resupinatum } \\
\text { Fabaceae (ISTE } 84 \text { 157) }\end{array}$ & Tifilotu & Aerial part & As fodder \\
\hline $\begin{array}{l}\text { Tussilago farfara L. } \\
\text { Asteraceae (ISTE } 84 \text { 086) }\end{array}$ & $\begin{array}{l}\text { Hindiba, Kınaçiçeği, } \\
\text { Kınaotu, Öksürükotu }\end{array}$ & Leaf & $\begin{array}{l}\text { Cooked with onion as meal, } \\
\text { boiled as salad }\end{array}$ \\
\hline $\begin{array}{l}\text { Typha latifolia L. } \\
\text { Typhaceae (ISTE } 84 \text { 128) }\end{array}$ & Saz & Aerial part & As decorative \\
\hline $\begin{array}{l}\text { Urtica dioica L. } \\
\text { Urticaceae (ISTE } 84 \text { 108) }\end{array}$ & $\begin{array}{l}\text { Isırgan, Isırganotu, } \\
\text { Sirgan, Sirgan }\end{array}$ & Aerial part & Cooked with rice as meal and for pastry \\
\hline & & Aerial part & As fuel \\
\hline $\begin{array}{l}\text { Verbascum specıosum Schrad. } \\
\text { Scrophulariaceae (ISTE } 84 \text { 189) }\end{array}$ & Ayılahanası, Kabalak & Whole plant & As a gun in children's play \\
\hline & & Leaf & As fodder for sheep and goat \\
\hline $\begin{array}{l}\text { Vicia sativa L. subsp. nigra (L.) Ehrh. var. nigra } \\
\text { Fabaceae (ISTE } 84 \text { 031) }\end{array}$ & Fi, Fiotu, Yabanifi & Aerial part & As fodder \\
\hline $\begin{array}{l}\text { Viola gracilis Sibth. \& Sm. } \\
\text { Violaceae (ISTE } 84 \text { 096) }\end{array}$ & Hercaimenekşe, Menekşe & Whole plant & As decorative \\
\hline \multirow{3}{*}{$\begin{array}{l}\text { Viscum album L. subsp. album } \\
\text { Loranthaceae (ISTE } 84 \text { 088) }\end{array}$} & \multirow{3}{*}{ Çakum, Ökse, Ökseotu, Yapışkanotu } & Fruit & As glue \\
\hline & & Leaf & As tea for bracing \\
\hline & & Fruit & Eaten fresh and as pickle \\
\hline \multirow[t]{3}{*}{$\begin{array}{l}\text { *Vitis vinifera L. } \\
\text { Vitaceae (ISTE } 84 \text { 182) }\end{array}$} & Asma, Üzüm & $\begin{array}{l}\text { Juice sap } \\
\text { (obtained from } \\
\text { broken branches) }\end{array}$ & For hair and skin care \\
\hline & & Leaf & $\begin{array}{c}\text { Cooked with onion, rice and } \\
\text { some spices as farci }\end{array}$ \\
\hline & & Seed & Boiled and eaten or eaten as pop corn \\
\hline \multirow{2}{*}{$\begin{array}{l}\text { *Zea mays L. subsp. mays } \\
\text { Poaceae (Observation) }\end{array}$} & Mısır & Stilus & As cigarette \\
\hline & & Aerial part & As fodder \\
\hline
\end{tabular}

\section{RESULTS AND DISCUSSION}

During this research, 145 specimens were collected from the area. According to the results of the identifications, 96 plant taxa belonging to 42 families have ethnobotanical usages. The ethnobotanical usages of plants are given in Table 1. Among these 96 plant taxa, 60 taxa (51 wild, 9 cultivated) are used as edibles and 64 taxa (51 wild, 13 cultivated) are used for different purposes. Several taxa were recorded as being used for more than one purpose. Photos of some plants which were taken from the study area are given in Figure 2.

In a number of cases, some of the taxa are known under the same local name. For instance Ranunculus constantinopolitanus, Cichorium intybus as "Sakızotu", Taraxacum scaturiginosum, Tussilago farfara, Cichorium intybus as "Hindiba", Convolvulus arvensis, Convolvulus betonicifolius subsp. betonicifolius as "Leksiotu", Cirsium creticum subsp. creticum, Datura stramonium as "Eşekdikeni", Lamium purpureum var. purpureum, Scrophularia scopolii var. scopolii as "Ballıbaba", Malva nicaeensis, Malva sylvestris as "Ebegömeç, Ebegümeci", Mentha longifolia subsp. typhoides var. typhoides, Mentha spicata subsp. spicata as "Nane, Yabaninane", Verbascum speciosum, Petasites hybridus as "Kabalak", Sambucus ebulus, Sambucus nigra as "Lor, Lüver, Piran, Sultan, Şahmelek", Rubus canescens var. canescens, Rubus sanctus as "Böğürtlen, Diken, Mora", Oenanthe pimpinelloides, Capsella bursa-pastoris as "Kazayağg" and Sonchus asper subsp. glaucescens, Taraxacum scaturiginosum as "Sütlüot".

Local people usually consume plants as edibles. These 60 edible plant taxa are distributed among 30 families and 58 genera. The most frequently used families are Asteraceae and Rosaceae (13,3\%), Lamiaceae (11,7 \%), Brassicaceae $(8,3 \%)$, Geraniaceae, Apiaceae, Moraceae and Liliaceae (5\%). The genera which is represented with the highest number of taxa among edible plants are: Geranium, Malva, Mentha, Morus, Rubus and Rumex. 
The mostly used parts of edible plants are leaf, young stem and fruit. Leaves are usually boiled and eaten as salad or cooked as a meal and cooked for pastry. Leaves of Arum italicum are boiled a few times firstly and then the boiled water is thrown away because of its alkaloid content.

Some plants of the Apiaceae family like Oenanthe pimpinelloides, Heracleum platytaenium and Chaerophyllum byzantinum are consumed as edible or fodder in İzmit. But food plants of the Apiaceae family contain a group of bioactive aliphatic $\mathrm{C}_{17}$-polyacetylenes. These polyacetylenes have shown to be highly toxic towards fungi, bacteria and mammalian cells and to display neurotoxic, antiinflammatory and anti-platelet-aggregatory effects and to be responsible for allergic skin reactions (10). Also some members of Apiaceae family are reported to cause photosensitization $(11,12)$, because of these reasons they have to be used carefully.

Local people also use plants for different purposes in İzmit. These 64 various useful plant taxa are distributed among 32 families and 56 genera. Among these plants, the genera which is represented with the highest number of taxa are: Convolvulus, Ruscus, Sambucus and Trifolium. The most frequently uses are; 19 taxa as fodder, 7 taxa as fuel, 6 taxa as tea, 5 taxa as broom, 5 taxa in the treatment of animal diseases and 3 taxa for colouring henna. Rhodendron ponticum subsp. ponticum and Castanea sativa are used as beeplant. Flowers of Rhodendron ponticum subsp. ponticum is used for getting "Deli Bal (Poisonous honey)". But this plant contains Grayanotoxin I (Andromedotoxin) and can cause poisoning (13). Also Helleborus orientalis, Pteridium aquilinum and Sorghum halepense var. halepense are used as fodder and they can cause poisoning in animals (14).

According to our results, there is 1 endemic plant; Lathyrus undulatus which is used as fodder by local people. The responsibility of researchers is to give informations to local people about endemic plants and their usages. This result gives us an important information to protect our natural habitat in the study area for long term. Ethnobotanical knowledge becomes widespread by immigration. The majority of the population in İzmit consists of people all around the Turkey and also immigrants from Balkans and Caucasus. Because of this, ethnobotanical knowledge is mostly gathered from different areas, so various informations have been found in İzmit.

The results of our study show that even in countrys that are situated in close proximity to metropolitan cities, the ethnobotanical usages of plants are still alive. And also documenting not only about medicinal plants but also edible plants and plants for different usages (fodder, fuel etc.) are necessary before the knowledge of these usages has been completely lost.

\section{ACKNOWLEDGMENT}

This research was financially supported by İstanbul University Research Fund (Project No: T-989/06102006). Thanks to all interviewers who participated in this study by providing information.

\section{İzmit'in faydalı ve besin bitkilerinin etnobotaniği}

ÖZET: İzmit ilçesinde yapılan bu etnobotanik çalışmada 145 adet bitki örneği toplanmıştır. Arazi çalışmalarında yöre halkıyla görüşülerek bitkilerin kullanım amaçları ve kullanılan kısımları gibi bilgiler derlenmiştir. Yapılan bu çalışma sonucunda 42 familyaya ait 96 taksonun etnobotanik kullanımı olduğu saptanmıştır. Bunlardan 60 (51 doğal, 9 kültür) takson gıda olarak ve 64 takson da (51 doğal, 13 kültür) çeşitli diğer amaçlar için kullanılmaktadır. Yapmış olduğumuz çalışma ile büyük şehirlere yakın yerleşim alanlarında bile bitkilerin etnobotanik kullanımlarının hala varolduğu görülmüştür.

ANAHTAR SÖZCÜKLER: Etnobotanik, Türkiye, İzmit

\section{REFERENCES}

1. Davis PH. Flora of Turkey and the East Aegean Islands Vol. 1-9. Edinburgh University Press, London. 1965-1985.

2. Davis PH, Mill RR, Tan K. Flora of Turkey and The East Aegean Islands Vol. 10 (Suplement I). Edinburgh University Press, London. 1988.

3. Güner A, Özhatay N, Ekim T, Başer KHC. Flora of Turkey and The East Aegean Islands Vol 11 (Supplement II). Edinburgh University Press, London. 2000.

4. Özhatay N, Kültür Ş. Check-list of additional taxa to the supplement flora of Turkey III. Turk J Bot 2006; 30: 281316.

5. Özhatay N, Kültür Ş, Aslan S. Check-list of additional taxa to the supplement flora of Turkey IV. Turk J Bot 2009; 33: 191-226.

6. Özhatay N, Kültür Ş, Gürdal MB. Check-list of additional taxa to the supplement flora of Turkey V. Turk J Bot 2011; 35: 589-624.

7. Baytop T. Therapy With Medicinal Plants In Turkey, Past and Present. Nobel Tip Bookstore Press, İstanbul. 1999.
8. Kizılarslan Ç. An Ethnobotanical Survey In The South Part of İzmit Gulf. MSc Thesis, İstanbul University, Department of Pharmaceutical Botany. 2008.

9. Tutin TG, Heywood VH, Burges NA, Moore DM, Valentine DH, Walters SM, Webb DA. Flora of Europaea Vol 1-5. Cambridge University Pres, London. 1964-1980.

10. Christensen LP, Brandt K. Bioactive polyacetylenes in food plants of the Apiaceae family: Occurrence, bioactivity and analysis. J Pharmaceut Biomed 2006; 41: 683-693.

11. Ebermann R, Alth G, Kreitner M, Kubin A. Natural products derived from plants as potential drugs for the photodynamic destruction of tumor cells. J Photochem Photobiol B-Biol 1996; 36: 95-97

12. Hudson JB, Towers GHN. Therapeutic potential of plant photosensitizers. Pharmac Ther 1991; 49: 181-222.

13. Sütlüpınar $N$, Mat $A$, Satganoğlu $Y$. Poisoning by toxic honey in Turkey. Arch Toxicol 1993; 67: 148-150.

14. Baytop $T$, Baytop $A$, Mat A, Sun S. Poisonous Plants In Turkey, Plant Poisoning and Treatment Methods. İstanbul University Press, İstanbul. 1989. 